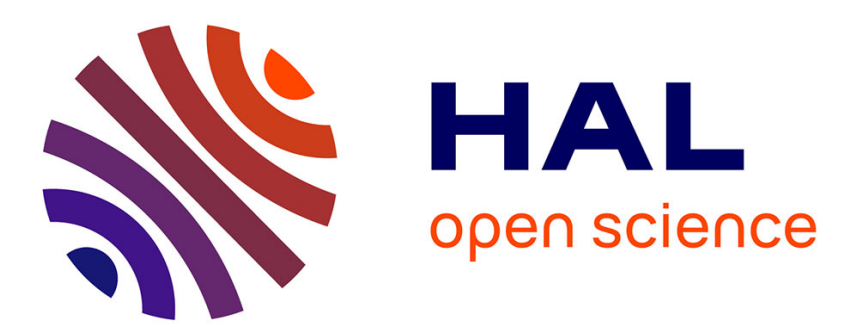

\title{
Relaxations diélectriques dans les polyéthylènes à très basses températures
}

\author{
J. Gilchrist, R. Isnard
}

\section{To cite this version:}

J. Gilchrist, R. Isnard. Relaxations diélectriques dans les polyéthylènes à très basses températures. Revue de Physique Appliquée, 1976, 11 (2), pp.245-252. 10.1051/rphysap:01976001102024500 . jpa00244054

\section{HAL Id: jpa-00244054 https://hal.science/jpa-00244054}

Submitted on 1 Jan 1976

HAL is a multi-disciplinary open access archive for the deposit and dissemination of scientific research documents, whether they are published or not. The documents may come from teaching and research institutions in France or abroad, or from public or private research centers.
L'archive ouverte pluridisciplinaire HAL, est destinée au dépôt et à la diffusion de documents scientifiques de niveau recherche, publiés ou non, émanant des établissements d'enseignement et de recherche français ou étrangers, des laboratoires publics ou privés. 
Classification

Physics Abstracts

8.740

\title{
RELAXATIONS DIÉLECTRIQUES DANS LES POLYÉTHYLẼNES A TRẼS BASSES TEMPÉRATURES
}

\author{
J. GILCHRIST et R. ISNARD (*) \\ Centre de Recherches sur les Très Basses Températures, C. N. R. S., \\ B. P. 166, 38042 Grenoble Cedex, France
}

(Reçu le 20 mai 1975, révisé le 23 juillet 1975, accepté le 29 septembre 1975)

\begin{abstract}
Résumé. - Nous avons observé les deux phénomènes de relaxation diélectrique qui se produisent dans les polyéthylènes aux températures de l'hélium liquide. Nous constatons que le phénomène qui a lieu à quelques kilohertz (relaxation de Vincett) est susceptible d'être étudié quantitativement avec un pont et nos résultats servent à confirmer les notions existantes, surtout en ce qui concerne l'effet d'oxydation. L'emploi de la méthode calorimétrique nous a permis d'observer la relaxation qui se produit à quelques mégahertz (relaxation de Carson), et d'apporter quelques éléments au débat sur l'origine de ce phénomène dont la constante de temps est assez bien définie.
\end{abstract}

\begin{abstract}
We have observed the two dielectric relaxation phenomena which occur in polyethylenes at liquid helium temperatures. We find that the phenomenon which takes place at several kilohertz (Vincett's relaxation) can be studied quantitatively with a bridge and our results confirm what is already known, particularly with regard to the effect of oxidation. Use of the calorimetric method has enabled us to observe the relaxation which occurs at several megahertz (Carson's relaxation), to note that its time constant is well defined and to make a few remarks about its origin.
\end{abstract}

1. Introduction. - Aux températures de l'hélium liquide les matériaux vitreux, y compris les polymères amorphes, ont un facteur (ou angle) de pertes diélectriques $(\delta)$ qui varie peu ou pas du tout en fonction de la température $(T)$ et de la fréquence $(f ; 2 \pi f \ll k T)$. Par contre, certains polymères qui manifestent un degré d'ordre cristallin, ou un ordre à courte distance, présentent des relaxations assez bien ou même très bien définies. Des relaxations dans les polyéthylènes et le poly (4-méthyl pentène 1) ont été observées ou étudiées depuis quelques années. Elles semblent résulter de la présence de groupements polaires incluant un proton qui peut se déplacer par effet tunnel. La concentration des dipôles qui participent aux relaxations est trop faible pour être détectée par les méthodes chimiques ou spectroscopiques habituelles, et la caractérisation des paramètres physiques des relaxations a été jusqu'ici une démarche plus fructueuse. Dans un cas il a été possible de déduire le moment dipolaire et dans un autre cas la distance parcourue par le proton. Ces résultats sont précisés dans les paragraphes qui suivent.

La poursuite de ces études nous semble profitable non seulement pour mieux connaître la nature des groupements dipolaires, mais aussi leur environnement, leur répartition et leurs interactions. Au cours de la

(*) Laboratoire d'Electrotechnique, Institut National Polytechnique de Grenoble. réalisation à cet effet d'un ensemble de dispositifs de mesure, nous avons étudié quelques polyéthylènes, et obtenu des résultats qui appellent quelques commentaires. Il nous a aussi paru utile de faire le point de la situation.

2. Résultats expérimentaux antérieurs. - Il existe de nombreuses études des pertes diélectriques dans les polyéthylènes aux basses températures. Les résultats sont souvent présentés sous formes de courbes $\delta(T)$ à une série de fréquences comprises entre $50 \mathrm{~Hz}$ et $50 \mathrm{kHz}$. A $T>200 \mathrm{~K}$ il y a peu de corrélations entre les courbes présentées par les différents auteurs, mais en dessous de cette température l'allure des courbes est mieux définie. Elle est caractérisée par une relaxation dite $\gamma(\grave{a} T=170 \pm 5 \mathrm{~K}$ pour $f=1 \mathrm{kHz}$ ) et une zone $(10 \mathrm{~K}<T<160 \mathrm{~K})$ où d'éventuels pics de relaxation sont peu intenses. Dans les cas $[1,2,3,4]$ où les courbes sont tracées à partir des températures de l'hélium liquide un phénomène est parfois décelé $[3,4]$ que nous désignons sous le nom de relaxation de Vincett ou pic $V$. Vincett [5] a mis au point un montage expérimental dont le principe est de mesurer, à l'aide de résistances de carbone, l'élévation de température d'une capacité à diélectrique solide lorsqu'elle est alimentée par une tension alternative connue. Il a ainsi trouvé sur un polyéthylène haute densité polymérisé par le procédé Phillips (appelons ce matériau HDP), des courbes isothermes $\delta(f)$ dont l'allure est celle de la 
relaxation de Debye. Par la suite plusieurs auteurs ont étudié plus systématiquement le même phénomène $[6,7$, $8,9,10]$ et ont contribué au développement d'un modèle théorique [6], [9] auquel nous reviendrons. Il a été démontré [6] que l'intensité, $A$, de la relaxation

$$
\left(A=\int_{f_{1}}^{f_{2}} f^{-1} \delta(f) \mathrm{d} f, \quad f_{1} \ll f_{\max } \ll f_{2}\right)
$$

diminue en fonction de $T$

$$
(A(4 \mathrm{~K}) / A(1 \mathrm{~K}) \sim 0,6 \text { typiquement })
$$

et en fonction de $E_{0}$, où $E_{0}$ est un champ continu intense superposé au champ alternatif de mesure. Le pic du facteur de pertes, $\delta_{\max }$, se produit à une fréquence $f_{\max }$ qui n'obéit pas à la loi d'Arrhenius mais varie comme $T$, sauf pour $T>3 \mathrm{~K}$ où la variation est plus rapide. $f_{\max }$ est indépendante de $E_{0}$.

Le phénomène est absent dans du polyéthylène sans additif, préparé de manière à éviter toute oxydation [10]. Il apparaît de façon peu intense après une faible oxydation, par exemple celle qui résulte de l'exposition à l'atmosphère au cours de la préparation ou du stockage. $\delta_{\max }$ prend une valeur de quelques microradians $(\mu)$. Différents traitements d'oxydation modérée accentuent le phénomène et on trouve $\delta_{\max } \sim 100 \mu$. Une relaxation peut aussi être observée dans du polyéthylène non oxydé contenant un additif antioxydant [10]. L'isotherme $\delta(f)$ présente alors une bosse plus large avec la même $f_{\max }$. Le polyéthylène oxydé a été étudié après certains traitements thermiques, chimiques et mécaniques [9]. Ainsi l'étirage du matériau ne modifie pas le phénomène mais l'absorption d'eau conduit à une diminution de l'intensité de la relaxation.

En plus du HDP, les polyéthylènes basse densité (BD) ont fait l'objet d'études moins systématiques. Ces matériaux sont caractérisés par un plus fort taux de branchements moléculaires et une moindre cristallinité. $f_{\text {max }}$ est toujours plus grande et dans un cas [6], $A(T)$ augmente entre $1,4 \mathrm{~K}$ et $4,2 \mathrm{~K}$.

L'extension des mesures vers les plus hautes fréquences a permis à Carson [9] d'étudier un second phénomène de relaxation (pic $C$ ) dans du HDP oxydé, et de l'observer dans du BD oxydé. Ce pic est plus intense que le pic $\mathrm{V}$ et la bosse est plus large (temps de relaxation moins bien défini). Les caractéristiques principales sont une variation linéaire de $f_{\max }(T)$ entre $1,9 \mathrm{~K}$ et $4,2 \mathrm{~K}$, peu de variation de $\delta_{\text {max }}(T)$ entre $11,5 \mathrm{~K}$ et $4,2 \mathrm{~K}$ (sauf un point aberrant à $1,5 \mathrm{~K}$ ) et la noninfluence du champ polarisant $E_{0}$. De plus, un traitement du HDP oxydé à la vapeur $\mathrm{D}_{2} \mathrm{O}$ entraîne la diminution du pic $\mathrm{C}$ préexistant et l'apparition d'un nouveau pic à une fréquence 150 fois plus basse, où les caractéristiques principales du pic $\mathrm{C}$ sont conservées, avec cette fois une légère décroissance de $\delta_{\max }(T)$. Le pic C préexistant est restauré lors d'un traitement à la vapeur $\mathrm{H}_{2} \mathrm{O}$.
3. Montages expérimentaux. - Nous avons utilisé deux systèmes de mesure, un pont Général Radio $1615 \mathrm{~A}$ avec son oscillateur et son amplificateur/détecteur, et un ensemble calorimétrique similaire à ceux mis au point par Vincett à Cambridge et par Phillips et al. à Stanford [7]. Dans les études antérieures les phénomènes ont été caractérisés à l'aide des résultats obtenus par la méthode calorimétrique. Cependant l'emploi d'un pont à transformateur [8] est plus simple et plus rapide, et convient mieux pour une étude systématique $\mathrm{du}$ pic $\mathrm{V}$ en fonction de traitements divers du polyéthylène. Il suffit de faire l'étalonnage à l'aide de la méthode calorimétrique. Pour cela nous comparons au pont les pertes de l'échantillon à celles d'un condensateur au PTFE de valeur voisine. Les condensateurs au PTFE présentent en effet le double avantage d'avoir de faibles pertes presque indépendantes de $f$ et de $T$ aux basses températures (vu par la méthode calorimétrique), et d'être constitués d'un matériau chimiquement stable. Pour les mesures avec le pont, les condensateurs sont placés dans une boîte immergée dans l'hélium liquide et remplie d'hélium gazeux sous pression réduite. Les mesures s'étendent de $20 \mathrm{~Hz}$ à $20 \mathrm{kHz}$ et de $1,3 \mathrm{~K}$ à $4,2 \mathrm{~K}$. Pour la méthode calorimétrique nous disposons d'un cryostat avec un double calorimètre, permettant de fonctionner entre $1,3 \mathrm{~K}$ et $6 \mathrm{~K}$ avec une gamme $f=5 \mathrm{~Hz}$ à $50 \mathrm{MHz}$. La performance de' notre dispositif est comparable à ceux dont il s'inspire $[5,7]$ Toutes nos mesures sont faites sans champ continu.

Nous avons toujours adopté la même technique de fabrication des capacités ainsi que le même matériau pour les électrodes, en nous appuyant sur les travaux précédents. Ainsi toutes les capacités sont en forme de double sandwich $[1,5]$ avec des électrodes en feuilles de plomb. Les deux électrodes basse tension (reliées au détecteur du pont ou à la masse pour la calorimétrie) sont de dimensions supérieures à l'électrode haute tension (reliée au transformateur-tête de pont ou à la source de signal). Les deux morceaux d'échantillon débordent de l'ensemble. Il n'y a pas d'anneau de garde. Lors de l'assemblage quelques gouttes de paraffine liquide sont déposées sur chaque surface de l'échantillon. L'ensemble est comprimé entre des plaques métalliques rigides à l'aide de ressorts feuillards.

L'emploi de la paraffine nécessite une explication. Vincett [5] l'a introduit pour assurer un meilleur contact thermique entre le diélectrique et les électrodes, mais en même temps il a minimisé les pertes parasites. Celles-ci sont de deux types : d'une part une série de pics correspondant aux résonances mécaniques de la capacité ou de ses composants et d'autre part un terme qui varie peu avec la fréquence, attribué à l'injection de charges [7]. Il ressort de l'étude faite à Stanford [7] que les électrodes en feuilles de plomb donnent lieu à des résonances nettement moins intenses que les électrodes en feuilles de cuivre. Nous confirmons cette observation. En ce qui concerne le deuxième type de parasite l'effet de la paraffine est obscur. Toutefois 
elle est de nature chimique semblable aux polyoléfines et remplit le vide. D'autre part il est généralement admis que les électrodes évaporées sont mauvaises, surtout aux basses températures où elles se décollent. L'étude des effets que nous qualifions de parasites pourrait se prolonger, mais pour le présent nous préférons rester à la constatation que tout effet parasite augmente la valeur apparente de $\delta$ et en général la rend dépendante de la tension d'essai. La meilleure capacité est celle pour laquelle $\delta$ est minimum et le moins dépendant de la tension. Signalons que celle-ci est de l'ordre de $200 f^{-1 / 2} \mathrm{~V}$ ( $f$ en $\mathrm{kHz}$ ) mais jamais plus de $800 \mathrm{~V}$ pour la calorimétrie telle que nous la pratiquons et $30 f \mathrm{~V}$, limitée à $20 \mathrm{~V}$, pour le pont. En général la variation de $\delta$ en fonction de la tension est modeste ou négligeable en dehors des résonances.

Vincett a pu s'assurer que sa paraffine ne contribue pas sensiblement, et de façon directe, aux pertes observées. Nous avons effectué un essai de vérification semblable, avec du feutre de polyéthylène sec (densité 0,41 ) et rempli de paraffine (densité 0,89). L'essai indique que la contribution à $\delta$ de notre paraffine pourrait être non négligeable, mais est indépendant de $f$ et de $T$. Cependant nous devons signaler que le polyéthylène BD absorbe la paraffine, ce qui peut provoquer un changement de sa morphologie, et influencer les phénomènes étudiés. Cette remarque intéresse aussi bien les résultats que nous allons présenter que ceux publiés antérieurement.

4. Echantillons. - Nos échantillons proviennent de deux lots de matériau : du BD distribué dans le cadre du Club Français d'études des Matériaux Macromoléculaires à l'état solide et reçu sous forme de gaine d'épaisseur $100 \mu \mathrm{m}$; de haute densité polymérisé à l'aide d'un catalyseur Ziegler Natta, que nous désignons HDZN, et que nous avons reçu moulé en plaque d'épaisseur $450 \mu \mathrm{m}$. Les deux lots ont été caractérisés, mis en forme et fournis par nos collègues du Laboratoire de Chimie Macromoléculaire de l'Université Claude Bernard de Lyon et en particulier M.P. Berticat.

BD : provenance S. N.P. A. ; $\left\langle M_{n}\right\rangle=56700$; densité 0,925 ; cristallinité $53 \%$; taux de branchement 20 méthyle $\%$ carbone ; pas de trace d'oxydation aux infra-rouges. Avant de l'étudier nous avons conservé ce matériau pendant neuf mois dans un vide primaire à l'abri de la lumière.

HDZN : provenance Naphtachimie (non purifié) ; $\left\langle M_{w}\right\rangle=170000$; coefficient de polymolécularité 9 ; densité 0,9715 ; cristallinité $82 \%$; taux de branchement 1 méthyle $\%$ carbone ; 0,3 carbonyle $\%$ carbone.

Trois échantillons découpés dans chaque lot de matériau se distinguent par des traitements d'oxydation quantitativement différents mais qualitativement similaires puisqu'il s'agit toujours d'une exposition à l'air sans lumière à $125^{\circ} \mathrm{C}$, pour une durée déterminée. L'oxydation est caractérisée par l'intensité de la bande d'absorption dans. l'infra-rouge à $5,8 \mu \mathrm{m}$. Cette bande est caractéristique des groupements carbonyle et carboxyle [11]. Nous l'attribuons entièrement aux carbonyles qui sont supposés prépondérer dans le cas d'oxydation à température élevée dans le noir [12].

5. Résultats. - Le premier échantillon $\mathrm{BD} 1$ a été étudié par la méthode calorimétrique directement après avoir été découpé dans le lot $\mathrm{BD}$. Il présente un pic $\mathrm{V}$ de quelques microradians. L'échantillon BD 2 a subi une oxydation à $125^{\circ} \mathrm{C}$ (état fondu) pendant $4 \mathrm{~h}$. Ses isothermes $\delta(f)$, obtenues avec le pont, sont portées sur la figure 1. Un autre échantillon BD 3 devait rece-

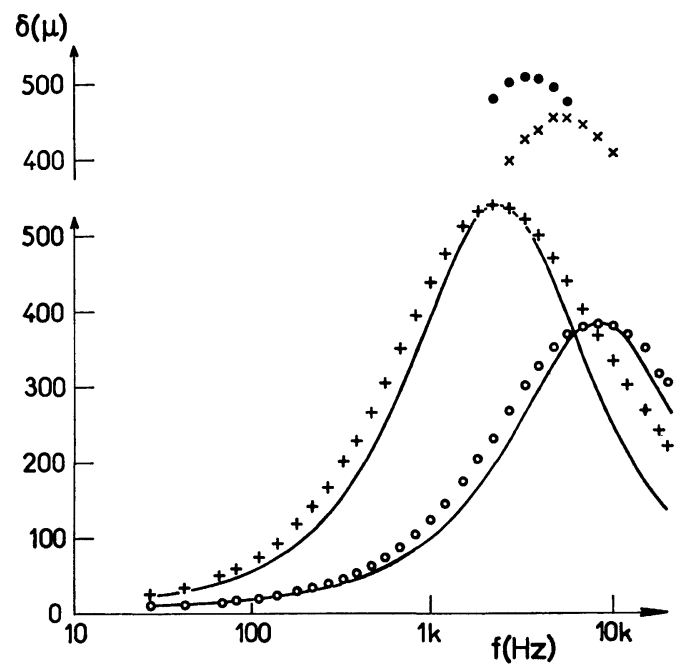

FIg. 1. - Facteur de pertes diélectriques d'un polyéthylène basse densité oxydé (échantillon BD 2) déterminé avec le pont à $4,2 \mathrm{~K}(O), 2,86 \mathrm{~K}(\times), 1,98 \mathrm{~K}(\bullet)$ et à $1,35 \mathrm{~K}(+)$. $\delta$ est exprimé en unités de $10^{-6}$ ou microradians. Les courbes représentent la loi de Debye.

voir le même traitement d'oxydation que BD 2 pour être étudié par la méthode calorimétrique. Or le four ayant été accidentellement détéglé pendant le traitement, la comparaison entre les courbes de la figure 1 et celle de la figure 2 (supérieure) doit être qualitative. Chaque fois le pic V est observé, et sa variation relative, $\delta_{\max }\left(T^{\prime}\right) / \delta_{\max }(T)$ est toujours à peu près la même. Par contre, l'absence apparente du pic C dans le BD 3 (Fig. 2 supérieure) signifie que $\delta_{\max }<10 \mu$ (mesuré au-

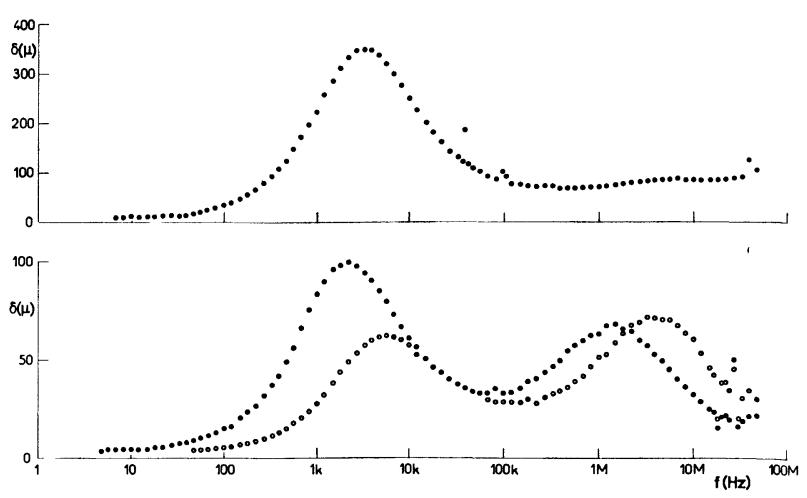

Fig. 2. - Facteurs de pertes diélectriques déterminés par la méthode calorimétrique. Partie supérieure : échantillon BD 3 à 2,01 K. Partie inférieure : échantillon HDZN 3 à 5,1 K (O) et à $2,02 \mathrm{~K}(\bullet)$. 
dessus du fond de $\delta \approx 70 \mu$ ), ou bien que le temps de relaxation est mal défini et la bosse est étalée sur plusieurs décades de fréquences.

Nous avons préparé trois échantillons également avec le polyéthylène haute densité. Le HDZN 1, simplement découpé et étudié sans oxydation volontaire, paraît avoir un pic $\mathrm{V}$ de valeur $20 \mu \pm 5 \mu$, mais ce résultat est peu précis parce que le condensateur était de $6 \mathrm{pF}$ au lieu des $60 \mathrm{pF}$ habituels. Nous voulions connaître la possibilité d'étudier d'autres diélectriques, dont la mise en forme est plus délicate. La présence du pic $\mathrm{V}$ dans le HDZN 1 pourrait être due aux impuretés, le matériau n'ayant pas été purifié. Ensuite nous avons préparé $\mathrm{HDZN} 2$ par un traitement de $4 \mathrm{~h}$ à $125^{\circ} \mathrm{C}$ (état solide). Les pics sont de $\delta_{\max }=93 \mu$ pour le $\mathrm{V}$ et $25 \mu$ pour le $\mathrm{C}$ à $2,02 \mathrm{~K}$. Par la suite nous avons réduit l'épaisseur de la plaque de $450 \mu \mathrm{m}$ à $90 \mu \mathrm{m}$ environ, par fusion entre deux plaques de quartz cristallin. Puis, la plaque mince a été exposée à l'air à $125^{\circ} \mathrm{C}$ (état solide) pendant $15 \mathrm{~h}$ et baptisée HDZN 3. Le matériau est alors devenu cassant et sa teneur en carbonyle est passée de $0,3 \%$ à $8 \%$ carbone. Ses isothermes $\delta(f)$, représentées sur la figure 2 (inférieure), manifestent clairement les deux pics avec des intensités voisines. Remarquons que l'intensité de la relaxation $\mathrm{C}$ semble augmenter lentement en fonction de $T$ entre $1,35 \mathrm{~K}$ et $5,1 \mathrm{~K}$, ainsi que le témoignent les courbes, mais cette augmentation pourrait être illusoire et provenir d'une erreur systématique dans la détermination de la tension aux bornes du condensateur. Toutefois, la variation est uniforme et il est exclu que $\delta_{\max }(T)$ décroisse. L'ensemble des observations de pics est résumé dans le tableau. Rappelons que l'intensité du pic V (mais non du pic C) diminue toujours en fonction de la température.

$\mathrm{Si}$ nous admettons que les polyéthylènes $\mathrm{HDZN}$ et HDP sont comparables, l'ensemble des résultats connus montre que sans oxydation ni certaines impuretés il n'y a pas de pic V ni de pic C. Sous l'effet d'oxydations progressives le pic $\mathrm{V}$ apparait le premier, son intensité augmente et puis se stabilise avec $\delta_{\max } \approx 100 \mu$ (à $4,2 \mathrm{~K}$ ). Le pic $\mathrm{C}$ apparaît après le $\mathrm{V}$ mais finit par dépasser celui-ci en intensité puisque $\delta_{\max } \approx 400 \mu$ [9] après une oxydation de $8 \mathrm{~h}$ dans l'air à $150^{\circ} \mathrm{C}$ (état fondu).

Aux environs de $50 \mathrm{~Hz}$ et de $4,2 \mathrm{~K}$ le facteur de pertes d'un polyéthylène est faible. Nous trouvons 10 à $12 \mu$ pour les BD oxydés et 4 à $5 \mu$ pour les HDZN oxydés. Pour les polyéthylènes non oxydés la valeur est généralement comprise entre $1,8 \mu$ et $6 \mu[5,10,13]$.

\section{TABleAU}

\begin{tabular}{cccrr} 
& Oxydation & & \multicolumn{3}{c}{$\delta_{\max }(\mu)$ à $2 \mathrm{~K}$} \\
Echantillon & à $125^{\circ} \mathrm{C}(\mathrm{h})$ & Carbonyles & \multicolumn{1}{c}{ pic V } & pic C \\
B - & - & - & - & - \\
BD 1 & 0 & $<0,1 \%$ & 7 & \\
BD 3 & 4 & $3 \%$ & 525 & \\
HDZN 1 & $<4$ & & 350 & $<10$ \\
HDZN 2 & 0 & $0,3 \%$ & 20 & \\
HDZN 3 & 4 & & 93 & 25 \\
& 15 & $8 \%$ & 99 & 68
\end{tabular}

6. Modèle de Phillips. - Nous décrivons ici le modèle développé par Phillips pour expliquer le pic $\mathrm{V}$ et utilisé par Carson à propos de son pic. La relaxation doit son origine à un ensemble de systèmes à deux niveaux d'énergie notés $\pm \Gamma$ (Phillips, $\pm \varepsilon$ ), et de moments dipolaires $\pm p$. A l'équilibre thermique la valeur moyenne du moment dipolaire est $p$ th $(\Gamma / k T)$. En présence d'un champ électrique $E, \Gamma$ devient $(\Gamma+p c E)$ où $c$ est le cosinus de l'angle entre l'axe du système (dipôle) et le champ. Lorsque la période du champ est petite devant le temps de relaxation des dipôles, les populations ne changent pas et nous trouvons pour la constante diélectrique :

$$
\varepsilon_{\text {adiabatique }}=\varepsilon_{\infty}+\sum c \frac{\partial p}{\partial E} \text { th }\left(\frac{\Gamma}{k T}\right) .
$$

Dans cette expression $\varepsilon_{\infty}$ est la constante diélectrique du milieu en l'absence des dipôles, et la sommation est étendue à tous les dipôles par unité de volume. Dans le cas d'une variation lente du champ :

$$
\varepsilon_{\text {isotherme }}=\varepsilon_{\infty}+\sum c \frac{\partial}{\partial E}\left[p \text { th }\left(\frac{\Gamma}{k T}\right)\right] .
$$

Il s'ensuit que l'intensité (supposée faible) de la relaxation est donnée par l'expression :

$$
\begin{aligned}
A & \approx \frac{\pi}{2 \varepsilon} \sum c\left\{\frac{\partial}{\partial E}\left[p \text { th }\left(\frac{\Gamma}{k T}\right)\right]-\frac{\partial p}{\partial E} \operatorname{th}\left(\frac{\Gamma}{k T}\right)\right\} \\
& =\frac{\pi}{2 \varepsilon k T} \sum c^{2} p^{2} \mathrm{ch}^{-2}\left(\frac{\Gamma}{k T}\right)
\end{aligned}
$$

où $\varepsilon \approx \varepsilon_{\text {isotherme }} \approx \varepsilon_{\text {adiabatique }}$. Cette expression est similaire à celle de la contribution à la chaleur spécifique de l'ensemble de systèmes à deux niveaux :

$$
C=k \sum\left(\frac{\Gamma}{k T}\right)^{2} \mathrm{ch}^{-2}\left(\frac{\Gamma}{k T}\right) .
$$

On sait que la connaissance de la chaleur spécifique sur une gamme de températures limitée ne permet pas de déterminer avec précision la densité d'états $n(\Gamma)$. Il en est de même pour $A(T)$.

Nous sommes invités à imaginer une coordonnée généralisée, $x$, qui caractérise la configuration d'un petit groupement d'atomes. L'énergie potentielle se présente sous la forme d'un double puits avec des minimums à $x= \pm l$, séparés par une barrière de hauteur $V$. La symétrie du puits est détruite par l'addition d'un gradient $\Delta / l$ de sorte que les deux minimums se différencient par $2 \Delta . l$ et $V$ sont les mêmes pour tous les doubles puits, tandis que $\Delta$ prend avec une même probabilité toutes les valeurs depuis 0 jusqu'à $\Delta_{1}$, c'està-dire :

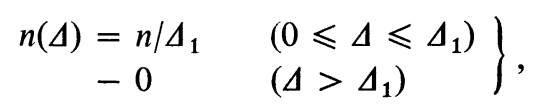

$n$ étant la concentration des dipôles. 
La théorie de l'hybridation des états permet d'écrire les relations :

$$
\begin{aligned}
\Gamma & =\left(\Delta^{2}+\Delta_{0}^{2}\right)^{1 / 2} \\
p & =(\Delta / \Gamma) p_{0}
\end{aligned}
$$

où $\Delta_{0}=a V \xi^{-1 / 2} \mathrm{e}^{-\gamma \xi}, \xi=\left(2 V m^{*} \hbar^{-2}\right)^{1 / 2} l$, $a$ et $\gamma$ sont des constantes d'ordre unité et $m^{*}$ est la masse réduite associée à la coordonnée généralisée. Les moments dipolaires $\pm p_{0}$ correspondent aux configurations $x= \pm l$ mais $p<p_{0}$ à cause de la localisation incomplète des états. $p_{0}$ et $\Delta_{0}$, tout comme $l$ et $V$, sont supposés identiques pour tous les puits. Il s'ensuit une densité d'états $n(\Gamma)$ comme celle de la partie supérieure de la figure 3 pour le cas $\Delta_{1} / \Delta_{0}=20$. La chaleur spéci-
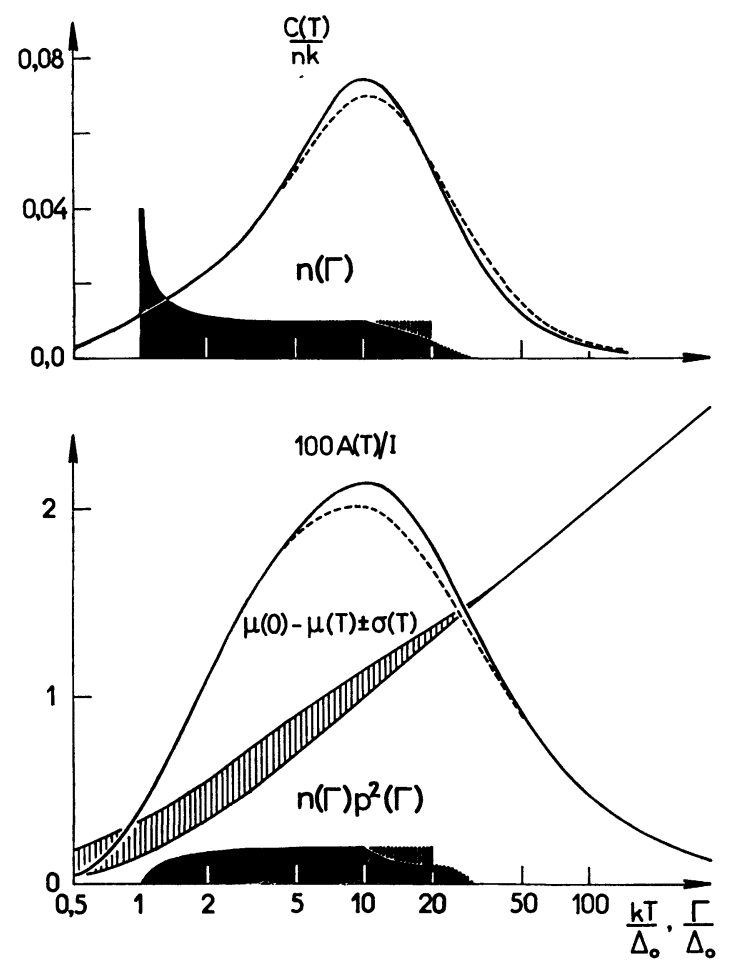

FIG. 3. - Illustration du modèle de Phillips. Partie supérieure : une densité d'états $n(\Gamma)$ (échelle arbitraire) avec la courbe de chaleur spécifique $C(T)$ correspondante (trait plein). Partie inférieure : densité d'états pondérée (échelle arbitraire) avec courbe d'intensité de la relaxation diélectrique $A(T)$ (trait plein) et courbes indiquant la variation du logarithme de la fréquence de la relaxation, et le manque de définition de celle-ci (explication dans le texte). Sur les deux parties de la figure, la température et le paramètre $\Gamma$ sont normalisés par rapport à une même constante $\Delta_{0}$. L'influence d'un champ polarisant $E_{0}=10 \Delta_{0} / p_{0}=0,5 \Delta_{1} / p_{0}$ est représentée par des hachures pour $n(\Gamma)$ et $n(\Gamma) p^{2}(\Gamma)$ et par les courbes en pointillés pour $C(T)$ et $A(T)$.

fique qui correspond à cette densité d'états a l'aspect d'une anomalie de Schottky élargie avec une plage de températures où $C \propto T$. Sur la partie inférieure de la figure est représentée la densité d'états pondérée $n(\Gamma) p^{2}(\Gamma)$ associée à $A(T)$. En mettant $c^{2}=\frac{1}{3}$ (pas d'orientation préférée) et en intégrant l'expression (3) on trouve la courbe $A(T) / I$, où $I=n p_{0}^{2} / \varepsilon \Delta_{0}$.
La représentation graphique $T A(T)$ nous paraît souhaitable pour essayer de déduire $\Delta_{0}$ et $\Delta_{1}$ à partir des données expérimentales $A(T)$, ou pour voir si une autre distribution $n(\Gamma)$ conviendrait mieux. Les expressions $T A(T)$ correspondant à plusieurs valeurs du rapport $\Delta_{1} / \Delta_{0}$ sont portées en coordonnées normalisées sur la figure 4 (supérieure), ainsi que d'autres courbes
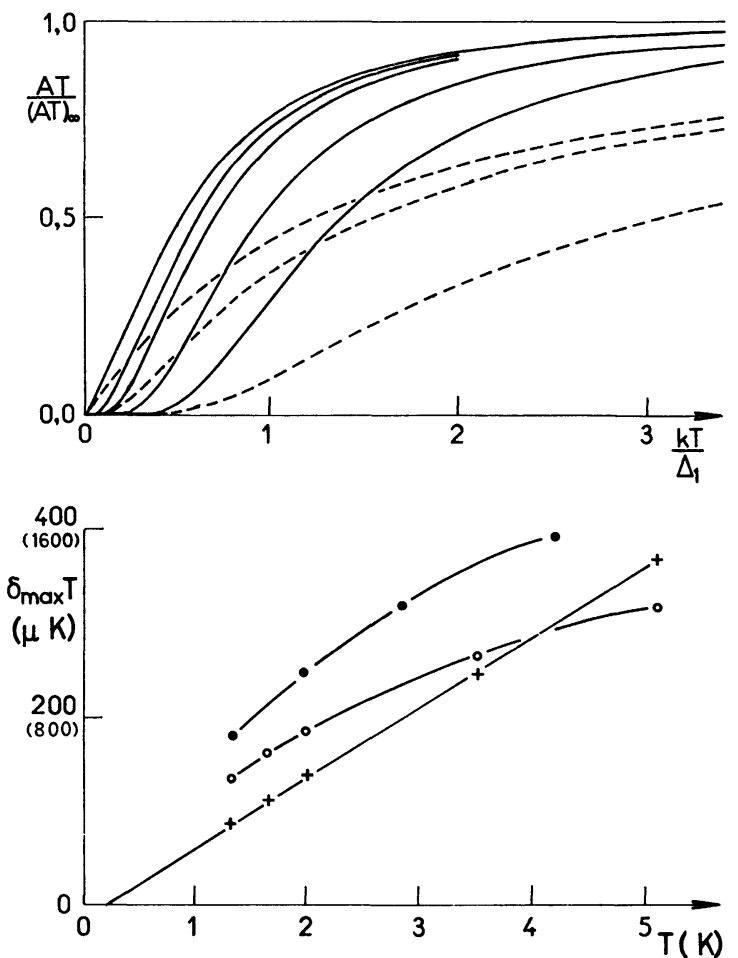

FIG. 4. - Etude des intensités des relaxations. Partie supérieure en courbes continues : prévisions du modèle de Phillips pour cinq valeurs du rapport $\Delta_{1} / \Delta_{0}(=\infty, 10,5,2,1$ de la plus haute à la plus basse). En courbes discontinues : une variante du modèle avec $\Delta_{1} / \Delta_{0}=\infty, 5,1$. Partie inférieure : nos résultats relatifs au pic C du HDZN 3 (+, échelle normale, de 0 à $400 \mu \mathrm{K}$ ), et au pic $\mathrm{V}$ du HDZN $3(\bigcirc$, échelle normale) et du BD $2(\bullet$, échelle réduite, de 0 à $1600 \mu \mathrm{K})$.

relatives à une variante du modèle de Phillips dans laquelle $n(\Gamma)$ a une queue asymptotique au lieu d'une fin abrupte : plus précisément

$$
n(\Delta)=\frac{2}{\pi} \frac{n}{\Delta_{1}} \frac{1}{1+\left(\Delta / \Delta_{1}\right)^{2}}
$$

remplace l'expression (5) et par conséquent :

$n(\Gamma) \sim\left[1+\left(\Gamma^{2}-\Delta_{0}^{2}\right) / \Delta_{1}^{2}\right]^{-1}\left(\Gamma^{2}-\Delta_{0}^{2}\right)^{-1 / 2} \Gamma$.

D'après les courbes nous pouvons obtenir des valeurs approximatives de $\Delta_{0}$ et $\Delta_{1}$ en regardant respectivement l'intersection avec l'axe des abscisses de la tangente à la courbe à son point d'inflexion ou sa partie droite, et l'épaulement de la courbe où sa courbure convexe est maximum.

Pour expliquer le processus de thermalisation des dipôles, Phillips emprunte la théorie de Sussmann [14]. Les transitions entre les états $\pm \Gamma$ s'accompagnent de 
l'absorption ou l'émission d'un phonon $2 \Gamma$. L'interaction a lieu parce qu'un champ de déformation $s_{i j}$ modifie la dissymétrie $\Delta$ par l'addition d'un terme $s_{i j} b_{i j}$. Ainsi à l'aide de la théorie des perturbations dépendant du temps, et en négligeant, pour simplifier, la nature tensorielle de la déformation, il trouve pour le temps de relaxation :

$$
\tau=\frac{\pi^{2}}{16} \frac{\hbar^{4} v_{\mathrm{s}}^{5} \rho}{b^{2} \Delta_{0}^{2} \Gamma} \operatorname{th}\left(\frac{\Gamma}{k T}\right)
$$

où $v_{\mathrm{s}}$ et $\rho$ sont la vitesse du son et la densité. D'après l'expression (3) les dipôles pour lesquels $\Gamma>k T$ contribuent peu à la relaxation. Pour les autres th $(\Gamma / k T) \approx \Gamma / k T$ avec comme conséquence des valeurs de $\tau$ peu sensibles à la valeur de $\Gamma$ et qui varient en $T^{-1}$. Le pic de relaxation est alors bien défini et $f_{\max }$ varie à peu près comme $T$. A partir des expressions (3), (5), (6), (7) et (10) nous avons calculé la valeur moyenne, $\mu$, et l'écart-type, $\sigma$, de $\log \tau$ pour le cas $\Delta_{1} / \Delta_{0}=20$. Les résultats sont représentés sur la figure 3 sous forme de courbes $\mu(0)-\mu(T) \pm \sigma(T)$, qui délimitent une zone hachurée. Malgré une certaine dispersion de valeurs de $\tau$ lorsque $k T \lesssim \Delta_{1}$, l'élargissement de la bosse de relaxation $\delta(\log f)$ est toujours inférieur à $4 \%$ et à peine appréciable. Il en est de même pour toute autre valeur $\operatorname{de} \Delta_{1} / \Delta_{0}$.

L'expression (10) est sensible à la valeur de $\Delta_{0}$, ce qui implique que celle-ci est assez bien définie, ainsi que les paramètres $l, m^{*}$ et $V$ dont elle dépend. Par contre, les seuls renseignements sur la valeur de $\Delta_{1}$ et de la forme de $n(\Delta)$ résultent de l'hypothèse [6], [14] selon laquelle $\Delta$, le paramètre de dissymétrie des puits, est associé à la déformation permanente du matériau, par le coefficient $b$. La déformation permanente est due à la contraction différentielle du matériau semi-cristallin, et $n(\Delta)$ donne une image des déformations existant localement. En tout cas les valeurs de $\Delta$ sont susceptibles d'être modifiées par la présence d'un champ électrique intense, ce qui entraîne une modification de $n(\Delta)$. Pour étudier l'effet il est préférable d'ajouter un champ continu, $E_{0}$, au champ alternatif qui reste relativement faible. On peut ainsi accéder à la valeur de $p_{0}$ si $n(\Delta)$ est déjà connu par des mesures sans $E_{0}$. C'est le cas du pic $\mathrm{V}$ pour lequel $p_{0} \approx 1,75$ Debyes. Sur la figure 3 est indiqué à titre d'exemple l'effet d'un champ $E_{0}=0,5 \Delta_{1} / p_{0}$, sur $n(\Gamma), C(T), n(\Gamma) p^{2}(\Gamma)$ (pondéré avec le facteur $c^{2}$ ) et $A(T)$.

L'accélération de la variation de $f_{\max }(T)$, observée pour le pic $\mathrm{V}$ quand $T>3 \mathrm{~K}$, est attribuée à une probabilité supplémentaire de transition due aux processus de second ordre du type diffusion inélastique d'un phonon. Les différentes possibilités ont été discutées $[14,15]$, et il a été noté [9] que si un état excité du système est impliqué (autre que $+\Gamma$ ), une information sur $V, m^{*}$ et $l$ est obtenue.

$\mathrm{Au}$ vu de l'ensemble des résultats il apparaît que les deux relaxations dans les polyéthylènes sont dues aux déplacements de protons sur une distance comparable à la maille atomique. Pour le pic $\mathrm{C}$, Carson propose la rotation d'une liaison longue de $0,11 \mathrm{~nm}$. En revanche la nature du groupement d'atomes est mal définie. On ignore s'il s'agit d'un hydroxyle, carboxyle ou peroxyle greffé sur la macromolécule, ou d'une molécule d'eau retenue près d'un tel groupement par liaison hydrogène. Toutefois la valeur de $p_{0}$ obtenue pour le pic $\mathrm{V}$ semble indiquer un groupement contenant un atome d'oxygène à l'exclusion d'une simple insaturation pour laquelle $p_{0} \sim 0,3$ à 0,5 Debye.

Enfin rappelons que le modèle de Phillips s'adapte aux matériaux amorphes en supposant que $\xi$, au lieu d'être bien défini, est plus ou moins distribué de la même façon que $\Delta$.

7. Quelques remarques. - Nous nous proposons de comparer les courbes théoriques $T A(T)$ à nos valeurs du produit $\delta_{\max } T$ de la figure 4 inférieure. L'évaluation de l'intensité est en effet rendu un peu arbitraire par le fond dissymétrique superposé aux bosses de relaxation, mais la forme des courbes $\delta(f)$ étant peu dépendante de la température, $A(T)$ doit varier un peu comme $\delta_{\max }(T)$. Les deux séries de points correspondant aux pics $\mathrm{V}$ présentent une courbure convexe et on peut déduire que $\Delta_{1} / k \approx 3 \mathrm{~K}$ pour chacun avec une légère préférence pour la variante (8) plutôt que l'expression (5). Il est à supposer que les points d'inflexion se situent en dessous de la température minimum atteinte, ce qui nous interdit d'estimer $\Delta_{0}$. En revanche les points correspondant au pic ' $\mathrm{C}$ dans le HDZN 3 sont bien alignés et s'extrapolent à $T=0,2 \mathrm{~K}$ pour $\delta_{\max } T=0$. Compte tenu de l'incertitude de notre résultat nous tirons la seule conclusion $\Delta_{0} / k<0,3 \mathrm{~K}$. L'absence de courbure interdit de déduire la valeur de $\Delta_{1}$ en dehors de l'inégalité

$$
\Delta_{1} / k>10 \mathrm{~K} \text {. }
$$

La figure 5 représente les fréquences $f_{\max }$ de nos différents pics. Elle confirme les observations précédentes. Remarquons d'abord l'absence de différence significative entre les trois $\mathrm{BD}$, à laquelle nous avons déjà fait allusion. Pour les pics $\mathrm{V}$ les points définissent une courbure positive, tandis que $f_{\max }(T)$ du pic $C$ est linéaire jusqu'à $5,1 \mathrm{~K}$ ce qui est en accord avec l'observation de Carson et indique l'absence ou le peu d'importance de processus de second ordre dans la relaxation $\mathbf{C}$. Pour l'interprétation dans le cadre du modèle de Phillips, cette même constatation est encore plus probante dans le cas du pic observé par Carson après le traitement au deutérium. On peut prévoir que le remplacement d'un proton par un deutéron accroît nettement la probabilité d'excitation thermique à un niveau supérieur à $+\Gamma$. Par exemple, si nous acceptons le postulat de Carson de la rotation (angle $\theta$ ) d'une liaison de $0,11 \mathrm{~nm}$ et de deux barrières $V / k=135 \mathrm{~K}$ (à $\theta=\pi / 2$, $3 \pi / 2$ ), nous trouvons [16] un état à $\delta / k \approx 71 \mathrm{~K}$ pour le proton et à $\delta / k \approx 58 \mathrm{~K}$ pour le deutéron. Ce dernier a une probabilité d'excitation appréciable à $4,2 \mathrm{~K}$ à partir de l'état $\pm \Gamma$, et une forte probabilité de se désexciter 


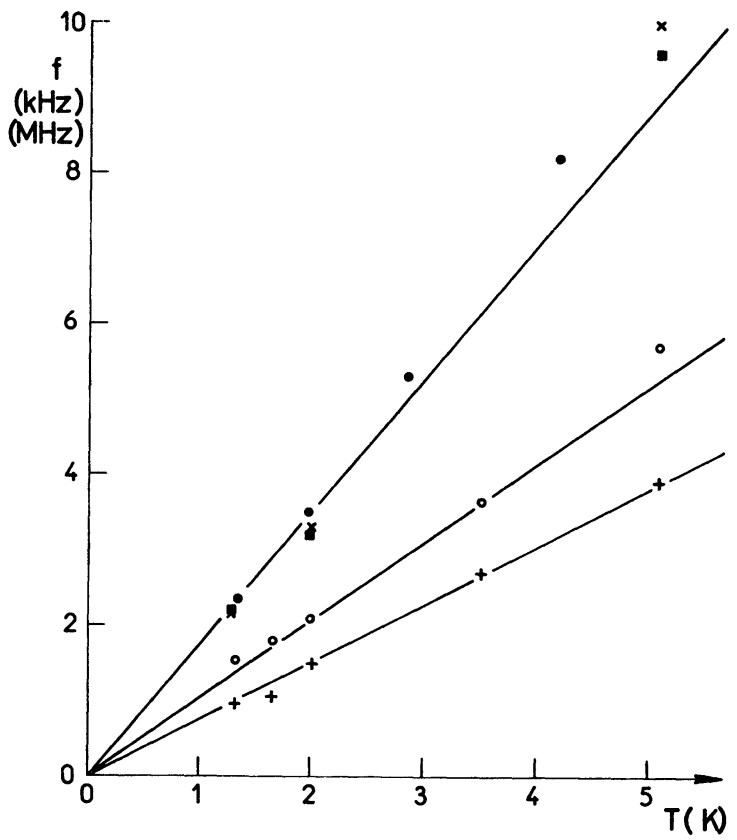

FIG. 5. - Etudes des fréquences des relaxations : pic $\mathrm{C}$ du HDZN $3(+, f$ en MHz), pic $\mathrm{V}$ du HDZN $3(O, f$ en $\mathrm{kHz})$, du BD $1(\square)$, du BD $2(\bullet)$ et du BD $3(\times, f$ toujours en $\mathrm{kHz})$. HDZN 3, BD 1 et BD 3 étudiés par méthode calorimétrique, BD 2 par pont.

vers l'autre état $\mp \Gamma$. Carson a vu la difficulté et pour éviter d'admettre un état excité encore plus bas il a adopté finalement les valeurs $\Delta_{0} / k \sim 0,6 \mathrm{~K}, \Delta_{1} / k \sim 4 \mathrm{~K}$. Nous constatons qu'une telle paire de valeurs ne convient pas pour le HDZN 3 d'après l'allure de $\delta_{\max }(T)$, et nous suggérons qu'il est peu probable que les valeurs soient tellement différentes du HDP au HDZN. C'est un des détails qui reste à éclaircir dans l'application du modèle théorique.

Dans le cas du pic $\mathrm{V}, p_{0}$ est connu [6], et on déduit qu'une relaxation Debye avec $\delta_{\max }=100 \mu$ à $4,2 \mathrm{~K}$ implique la participation de $0,5 \mathrm{ppm}$ de dipôles $(0,5 \mathrm{par}$ million d'atomes de carbone). Les mesures diélectriques permettent en principe de détecter $10^{-3} \mathrm{ppm}$. Cependant le rapport entre le nombre de dipôles actifs dans la relaxation et le nombre de groupements chimiques du même type est inconnu. Par exemple il est possible que seulement $1 \%$ des groupements participent, mais la concentration globale est toujours trop faible pour être détectée aux infra-rouges. Desétudes [9] avec des alcools et des acides démontrent qu'il ne suffit pas d'avoiı des $-\mathrm{OH}$ dans une matrice de molécules linéaires d'hydrocarbone. Cette observation conduit à l'hypothèse que les dipôles actifs sont ceux qui occupent des sites bien particuliers dans la structure cristalline, par exemple aux repliements de chaîne [9]. Nous suggérons que la valeur typique de $0,5 \mathrm{ppm}$ pour le pic $\mathrm{V}$ dans les hautes densités correspond au nombre de tels sites. Remarquons que si les dipôles actifs étaient uniformément répartis dans le matériau (hypothèse peu probable) leurs interactions électriques seraient faibles: $n p^{2} / \varepsilon k \sim 2,5 \mathrm{mK}$. Par contre quand la concentration, $n^{\prime}$, de carbonyle (moment $p^{\prime}$ ) est de l'ordre de $3 \%$ le milieu est loin d'être diélectriquement neutre, puisque $n^{\prime} p p^{\prime} / \varepsilon k \sim 25 \mathrm{~K}$. En plus il convient de rappeler que pour chaque carbonyle créé par oxydation il sera créé une molécule d'eau. Celle-ci peut être retenue dans le polyéthylène par liaison hydrogène.

Deux remarques s'imposent sur la possibilité d'autres manifestations physiques des systèmes à deux niveaux, et en particulier de ceux qui participent au pic $\mathrm{V}$. D'abord si $n=0,5 \mathrm{ppm}$ et $\Delta_{1} / k=3 \mathrm{~K}$ leur contribution à la chaleur spécifique est de $\sim 7 \times 10^{-7} \mathrm{~T} \mathrm{~J} /$ mole.degré ${ }^{2}$ soit assez modeste pour $1 \mathrm{~K}<T<3 \mathrm{~K}$, mais comparable à la partie qui varie en $T^{3}$

$$
\left(\sim 2 \times 10^{-4} T^{3} \mathrm{~J} / \text { mole.degré }{ }^{4}[17]\right)
$$

extrapolée à $T=0,2 \mathrm{~K}$. Ensuite, Phillips [6] donne une valeur du potentiel de déformation qui entre dans l'expression (10) $: b / k \approx 50 \mathrm{~K}$. Cèci permet de prévoir l'ordre de grandeur de l'intensité de la relaxation mécanique. L'expression analogue à (3) est :

$$
A_{\text {mec }}=\frac{\pi}{2 c k T} \sum b^{2} \mathrm{ch}^{-2}\left(\frac{\Gamma}{k T}\right),
$$

où $c=\rho v_{\mathrm{s}}^{2} \sim 4 \times 10^{9} \mathrm{~J} / \mathrm{m}^{3}$ et $A_{\text {mec }}$, comme $A$, est l'intensité relative, sans dimension. A $T=2 \mathrm{~K}$, $A_{\text {mec }} \sim 3 \times 10^{-8}$ cel qui est trop faible pour être détectée. Remarquons que la relaxation de systèmes à deux niveaux semble déterminer l'atténuation sonore dans la silice vitreuse dans certaines conditions, mais alors $b / k \sim 10^{4} \mathrm{~K}[18]$ et le facteur $b^{2}$ est de $10^{4}$ fois plus grand.

8. Conclusions. - Résumons ce qui est nouveau dans nos résultats expérimentaux :

- Nous constatons que pour une étude quantitative et systématique de la relaxation $\mathrm{V}$ dans le polyéthylène un pont commercial est utilisable, et cinq fois plus rapide que la méthode calorimétrique utilisée antérieurement.

- Le pic de relaxation $\mathrm{C}$ n'est pas nécessairement plus large que le pic $\mathrm{V}$, et il peut correspondre à un temps de relaxation aussi bien défini. Contrairement au pic $\mathrm{V}$ son intensité ne décroît pas en fonction de la température entre $1,35 \mathrm{~K}$ et $5,1 \mathrm{~K}$.

- Apparemment un polyéthylène haute densité Ziegler Natta se comporte de la même manière qu'un HDP (pour les deux pics), ce qui indique que d'éventuels résidus de catalyseur jouent un rôle secondaire. Par contre divers polyéthylènes basse densité semblent se comporter différemment et l'origine des différences reste à éclaircir.

- Pour une faible dose d'oxydation le pic $\mathrm{V}$ est plus intense que le pic $\mathrm{C}$, mais pour une dose plus forte l'inverse est vrai. Dans les hautes densités il semble se produire un effet de saturation du pic $\mathrm{V}$ à une intensité de l'ordre de $\delta_{\max } \approx 10^{-4}$. 
- Au vu de la teneur en carbonyles de nos échantillons, les dipôles qui participent aux différentes relaxations dans les polyéthylènes oxydés ne constituent qu'une faible fraction des dipôles présents $\left(10^{-4}\right.$ par exemple). Les polyéthylènes bien oxydés sont loin d'être des matrices diélectriquement neutres.
Si le modèle de Phillips a permis de donner une description physique des deux phénomènes à part certains détails, des questions restent à résoudre sur la nature des dipôles et leur emplacement, et sur la symétrie cristalline, ou à courte distance, nécessaire pour que le temps de relaxation soit bien défini.

\section{Bibliographie}

[1] Chant, M. J., Cryogenics 7 (1967) 351.

[2] Allan, R. N. et Kuffel, E., Proc. IEEE 115 (1968) 432.

[3] Fallou, B. et Bово, J. C., Beama Electrical Insulation Conference (1971) compte rendu p. 197.

[4] IsNARD, R., thèse (1974), Université Scientifique et Médicale de Grenoble.

[5] Vincett, P. S., J. Phys. D 2 (1969) 699.

[6] Phillips, W. A., Proc. R. Soc. A 319 (1970) 565.

[7] Phillips, W. A., King, C. N., Thomas, R. A. et NorTON, R. H., Microwave Laboratory Report (non publié), $n^{\circ} 2176$ des W. W. Hansen Laboratories of Physics, Stanford University, 1973.

[8] Müller, F. H., Heybey, O. et Knispel, G., Kolloid Z. u. $Z$. Polym. 251 (1973) 932.
[9] Carson, R. A. J., Proc. R. Soc. A 332 (1973) 255.

[10] Thomas, R. A. et King, C. N., Appl. Phys. Lett. 26 (1975) 406.

[11] Heacock, J., Mallory, F. B. et Gay, F. P., J. Polym. Sci. 6 (1968) 2921.

[12] Rugg, F. M., Smith, J. J. et Bacon, R. C., J. Polym. Sci. 13 (1954) 535.

[13] Nelson, R. L., Proc. IEEE 121 (1974) 764.

[14] Sussmann, J. A., J. Phys. \& Chem. Solids 28 (1967) 1643.

[15] Sussmann, J. A., Phys. Matière Condensée 2 (1964) 146.

[16] Pauling, L., Phys. Rev. 36 (1930) 430.

[17] Tucker, J. E. et Reese, W., J. Chem. Phys. 46 (1967) 1388.

[18] JACKLE, J., Z. Phys. 257 (1972) 212. 